\title{
Stable nano-silver colloid production via Laser Ablation Synthesis in Solution (LASiS) under laminar recirculatory flow
}

\author{
B. Freeland ${ }^{1,2,3}$, R. McCann ${ }^{1,2,4, a)}$, G. Alkan ${ }^{5}$, B. Friedrich ${ }^{5}$, G. Foley ${ }^{1,3}$ and D. Brabazon ${ }^{1,2,4}$ \\ ${ }^{1}$ Advanced Processing Technology Research Centre, School of Mechanical \& Manufacturing \\ Engineering, Dublin City University, Glasnevin, Dublin 9, Ireland \\ ${ }^{2}$ I-Form Advanced Manufacturing Centre, Dublin City University, Glasnevin, Dublin 9, Ireland \\ ${ }^{3}$ School of Biotechnology, Dublin City University, Ireland \\ ${ }^{4}$ National Centre for Plasma Science and Technology, Glasnevin, Dublin 9, Ireland \\ ${ }^{5}$ Institute of Process Metallurgy and Metal Recycling (IME), RWTH Aachen University, Intzestraße 3, \\ Aachen 52056, Germany
}

a) ronan.mccann@i-form.ie

Keywords: Nanoparticle, silver, size-quenching, laser ablation synthesis in solution.

\begin{abstract}
As nanomaterials find applications in an increasingly diverse range of fields such as wastewater treatment, biotechnology and flexible electronics, the demand for nanomaterials with specific properties has increased. This increase is coupled with an increasing emphasis on nanomaterials with highly-specific properties for specialized applications. Industrially, nanomaterials are produced via wet-chemical techniques which employ the use of solvents and reagents which are environmentally harmful. As we move forward with the use of nanomaterials, the ability to produce nanomaterials in a sustainable manner has become a topic of great significance. Towards this end, Laser Ablation Synthesis in Solution (LASiS) is a physical production technique capable of producing tailored nanomaterial colloids in a sustainable manner. These colloids are produced by ablating a solid target immersed in a solvent using a laser. Typically, LASiS is conducted in a batch process and in small volumes limiting commercial viability. To overcome this, there has been a move towards the use of continuous production via LASiS using flow systems. This allows an increase in nanomaterial yield, resulting in colloids concentrations approaching those of commercial colloids. This work investigates a new production technique incorporating a laminar recirculatory flow system to produce stable high concentration nano-silver colloids.
\end{abstract}

\section{Introduction}

Silver nanoparticles (AgNps) are among the most widely studied and utilised nanomaterials in the world due to their uptake in applications across fields of electrical engineering, biotechnology and healthcare (1-4). However the established chemical synthesis nanomaterial production technique has a negative environmental impact due in part to its waste by-products. Furthermore, post-production washing steps may be need to remove ligands depending on the surface purity requirements of the application (5). Polymers bound on the nanoparticle surface can also be present post-production and they have been seen to detrimentally effect the conductivity of nano colloid printed circuits (2) . To combat these issues a "green-synthesis" technique has been developed over the past fifteen years. This technique utilises laser irradiation to ablate a solid material target submerged within a liquid, ejecting $\mathrm{Nps}$ from the plasma plume into the surrounding liquid, allowing for ligand free $\mathrm{Np}$ production and the potential for single step functionalisation. The emergence of LASiS can significantly reduce the amount of post-processing required when compared with chemical production. 
However, there are drawbacks with the LASiS technique where high-cost laser systems

\section{Experimental setup}

Silver nano-colloids were manufactured utilising a "semi-batch" Laser Ablation Synthesis in Solution (LASiS) technique, described in Figure 1. The developed approach is unique in terms of other LASiS "semi-batch" processes, such as the one described by Nachev et al. (18), as it ensures uniform laminar flow at the ablation site. The irradiation source used in this work was a picosecond-pulsed WEDGE HF 1064 (BrightSolutions, Italy) Nd:YAG laser with a wavelength of $1064 \mathrm{~nm}$, maximum output power of $1.2 \mathrm{~W}$, a pulse width of $700 \mathrm{ps}$ and a pulse repetition rate of $10 \mathrm{kHz}$.

The target material selected was silver plate $(>99.999 \%$ metals basis, Scottsdale bullion silver) machined and mechanically polished to produce discs of $8 \mathrm{~mm}$ outer diameter and 4 $\mathrm{mm}$ thickness. The target was placed into a custom designed 3-D printed flow cell. De-gassed, de-ionized water was employed as the base colloid solvent for most of the tests, with the addition of $0.86 \mathrm{mg} / \mathrm{L} \mathrm{NaCl}$ for additional colloid stability tests. The liquid was recirculated continuously over the surface of the target at a rate of $100 \mathrm{~mL} / \mathrm{min}$ during the tests. The high flow rate solvent regime ensured that efficient $\mathrm{Np}$ production was carried out, enabling the removal of possible masking effects from the laser plume, cavitation bubbles or Nps at the ablation site. The colloid was recirculated within a PTFE piping loop (total volume $100 \mathrm{ml}$ ) until the end of the test. This setup allowed the production of colloid under the set processing condtions. The laser beam's spot size was controlled at $140 \mu \mathrm{m}$ and operational fluence was 
maintained at $1.83 \mathrm{~J} / \mathrm{cm}^{2}$. The beam was scanned in an Archimedean spiral across the target surface at a speed of $2 \mathrm{~mm} / \mathrm{s}$. Flow control was performed via automatic valve and peristaltic pump actuation via a developed LabVIEW control program. Productions tests were carried out each with a set duration of 15 minutes each.

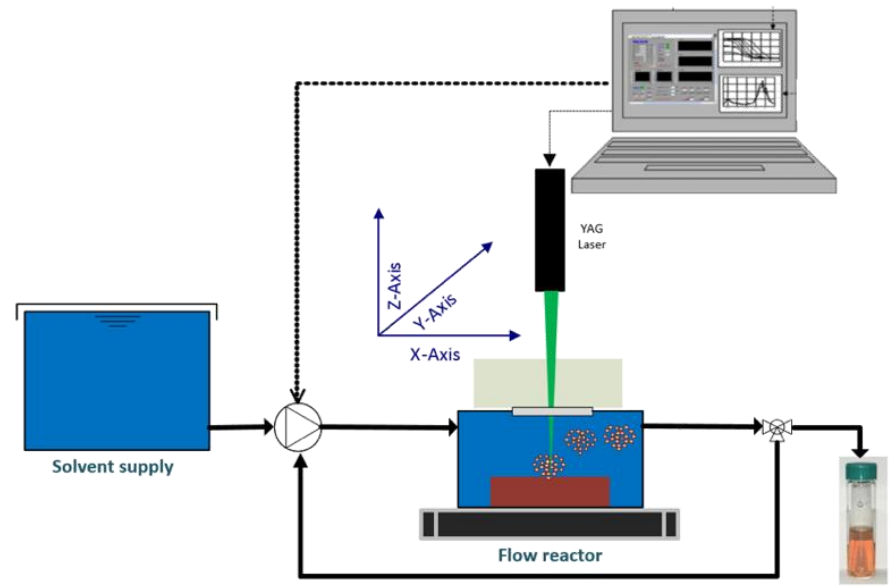

Figure 1: Schematic of the LASiS production setup including flow reactor cell containing the target material, solvent reservoir, laser system and control valves.

\section{Metrology}

The colloids size and morphology were determined with various analytical techniques. Np size distribution was determined via $180^{\circ}$ backscatter dynamic light scattering (DLS) and transmission election microscopy (TEM). The DLS used was a NanoFlex (Microtrac), with analysis performed on $2 \mathrm{~mL}$ samples. A $60 \mathrm{~s}$ acquisition time was used averaging over a set of five measurements. Background correction was applied with the base colloid solvent, in this cases DI-water. The TEM used was an FEI Titan with Field Emission Gun and spherical aberration corrector system (Cs-corrector) of the objective lens operated at $300 \mathrm{kV}$. Analysis was performed using a copper mesh TEM grid with $40 \mu \mathrm{L}$ of sample applied and allowed to evaporate at room temperature.

The Np colloids optical properties were characterized using a Biochrom Libra S22 UV/Vis spectrophotometer, with a scanning range of $200-900 \mathrm{~nm}$ with DI water background correction applied. For both DLS and UV-Vis measurements, a one-in-ten dilution of the colloid was used to mitigate measurement saturation, no post-filtration was performed, ensuring that the DLS and UV-Vis characterizations were representative of the production process.

A commercial silver Nps colloid (Sigma Aldrich, $10 \mathrm{~nm}, 25 \mathrm{ml}, 0.02 \mathrm{mg} / \mathrm{ml}$, SPR $403 \mathrm{~nm}$, \#730785) was compared against the produced colloids. The commercial colloid as measured via the same DLS and UV-Vis method as a calibration concentration reference.

\section{Results and Discussion}

The nano-colloid produced under continuous re-circulation conditions compares well with commercially available products prepared via wet chemical synthesis in terms of optical response, concentration and size dispersion. The DLS measurement observed a population size distribution peak at $21 \mathrm{~nm}$, with the distribution ranging from $10-50 \mathrm{~nm}$, see Fig. 2a. TEM imagining (see result in Fig. 2b) was in agreement with the hydrodynamic size distribution measured via DLS, noted in Fig. 2a. 


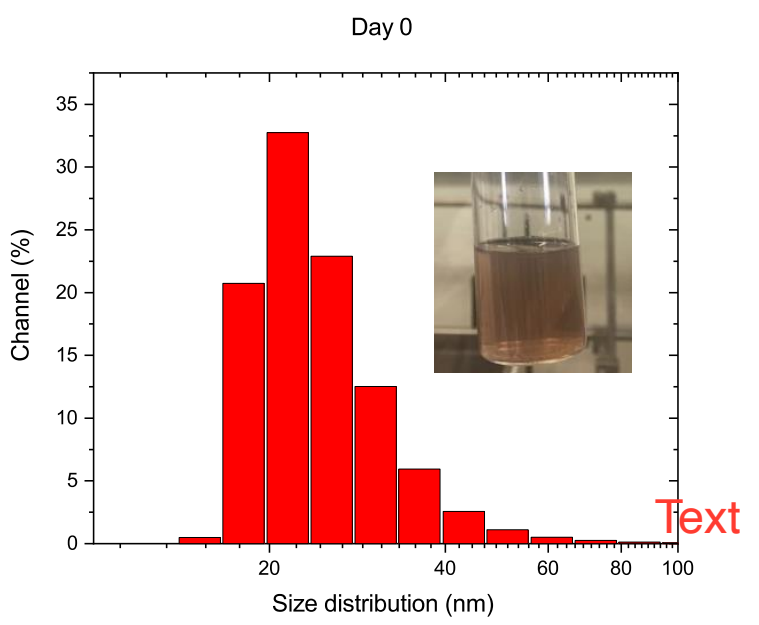

(a)

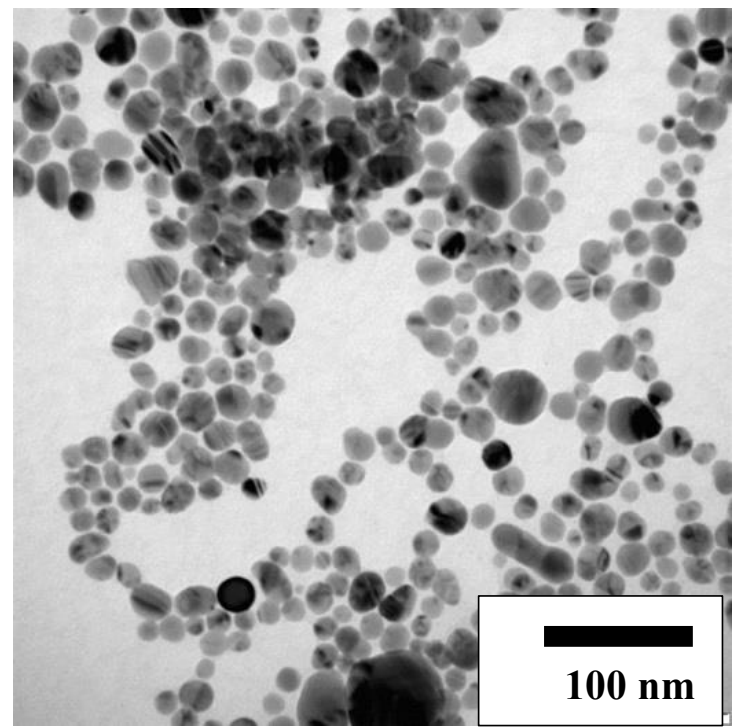

(b)

Figure 2: (a) DLS Np size distribution analysis, and (b) TEM image of AgNPs with scale bar of $100 \mathrm{~nm}$.

TEM imagining indicated a small population of larger particles $(>50 \mathrm{~nm})$ not observed in the DLS analysis. These particles may be due to aggregation during the TEM grid drop-casting step, or alternatively sedimented during DLS measurement. This alludes to the continuing need for a secondary, direct measurement technique such as TEM when characterizing Nps. The colloids displayed a surface plasmon resonance (SPR) band at $396 \mathrm{~nm}$ (Fig. 3) with a red shift to $402 \mathrm{~nm}$ after twelve days stored at room temperature. This spectrum compares well with commercial AgNps with an SPR at $403 \mathrm{~nm}$ and those reported in literature for laser produced Nps (19), (20).

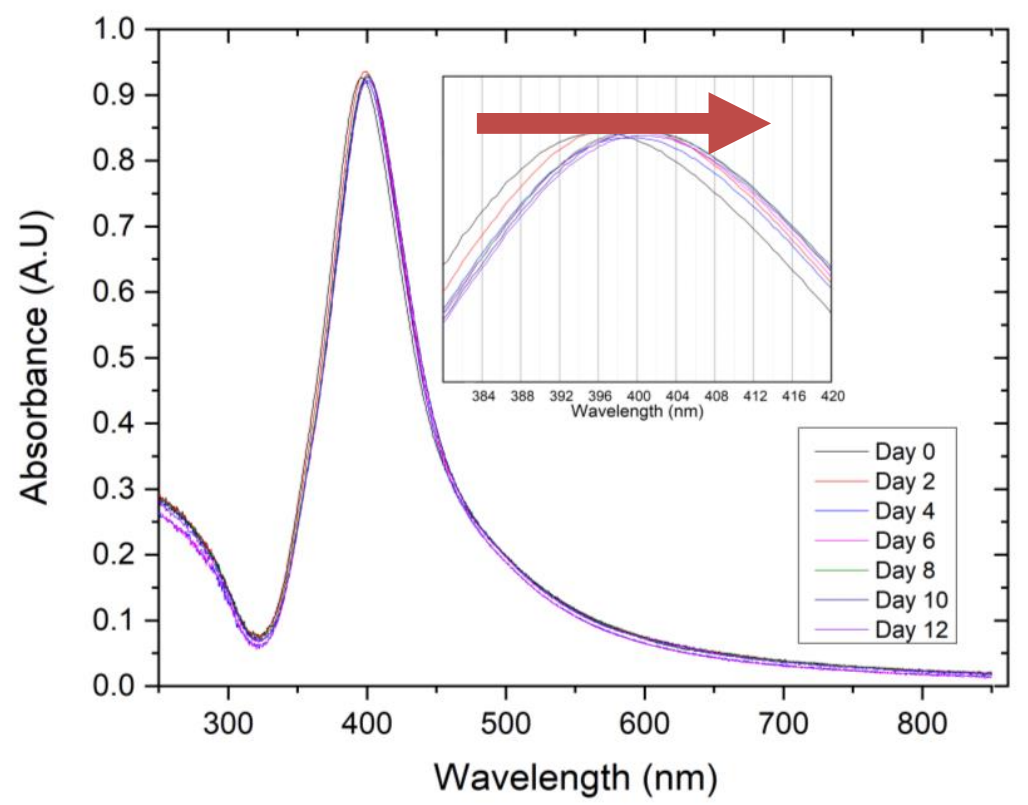

Figure 3: Absorption spectrum of AgNps produced, with a plasmonic peak at $396 \mathrm{~nm}$ at day

0 , and red shifting towards $402 \mathrm{~nm}$ at day 12 while showing a small intensity drop. 


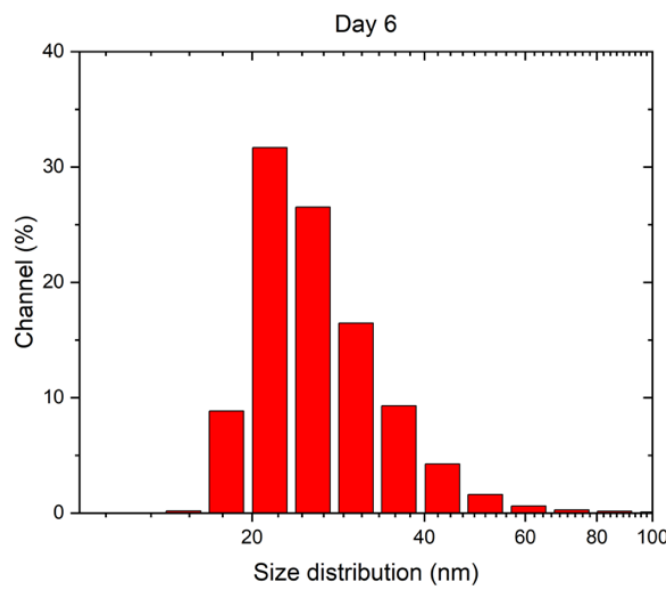

(a)

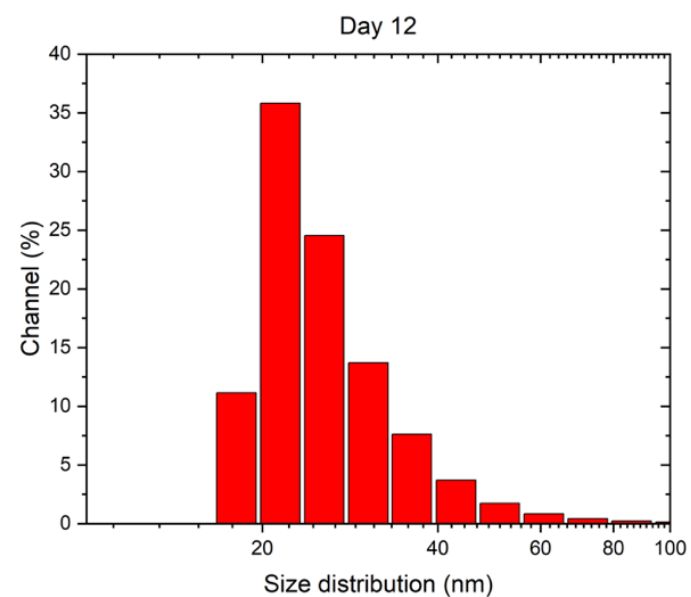

(b)

Figure 4: DLS analysis of AgNps colloid (a) after six days resting at room temperature, the peak diameter is $22.7 \mathrm{~nm}$ and distribution width (instrument defined variable, $2 \sigma$ ) is $11 \mathrm{~nm}$.

After twelve days (b) the indicated distribution change is within the accuracy of the measurement device; with a peak diameter of $22 \mathrm{~nm}$ and width of $12 \mathrm{~nm},(\mathrm{n}=3$,$) .$

\section{Electrolytes to aid size reduction}

It has been well established that the presence of electrolytes in the ablation liquid can have a size quenching effect on particle formation (23). The $\mathrm{NaCl}$ acts as a stabiliser on initial particles formed within the cavitation bubble. These "primary-particles" are smaller than the general colloid population (sub-10 nm) (24), as they can act as "seeding particles' for Np growth. Therefore if the "primary-particles" are stabilized, the colloid size distribution should exhibit a higher proportion of smaller Nps while lowering the possibility of agglomeration (5). Other studies have suggested that at low concentrations, $\mathrm{NaCl}$ addition can aid $\mathrm{Np}$ long-term stability (25), but at higher concentrations act as a destabilizer. In these tests, AgNps formation was tested in the presence of a low concentration ionic liquid environment.

In Fig. 5 a $3 \mathrm{~nm}$ red-shift on the SPR can be observed from day 0 to day 12 however the intensity dropped by a higher proportion (4.3\%) than the salt free AgNps. As this drop is 
approaching the limit of 5\% allowed deviation of absorbance, it would be expected that the colloid would be unstable over a period of 5 or more weeks (26). However size quenching did occur as the peak diameter has reduced from $22 \mathrm{~nm}$ when DI water was used to $11 \mathrm{~nm}$ when the weak ionic fluid was used. Therefore the application of ionic fluids allowed the fabrication of AgNps with equal Nps peak diameters to a commercially available example $(10 \mathrm{~nm})$. A smaller size distribution of only $5 \mathrm{~nm}$ at day 0 was recorded using the weak ionic solution compared to a main peak size distribution of about $12 \mathrm{~nm}$ when DI water was used. These results indicate that the AgNps size remained constant over the testing period but some colloid may have fallen out of suspension, leading to the drop in absorption intensity. This work shows promising results for using varying salts concentrations to adjust AgNps size distribution.

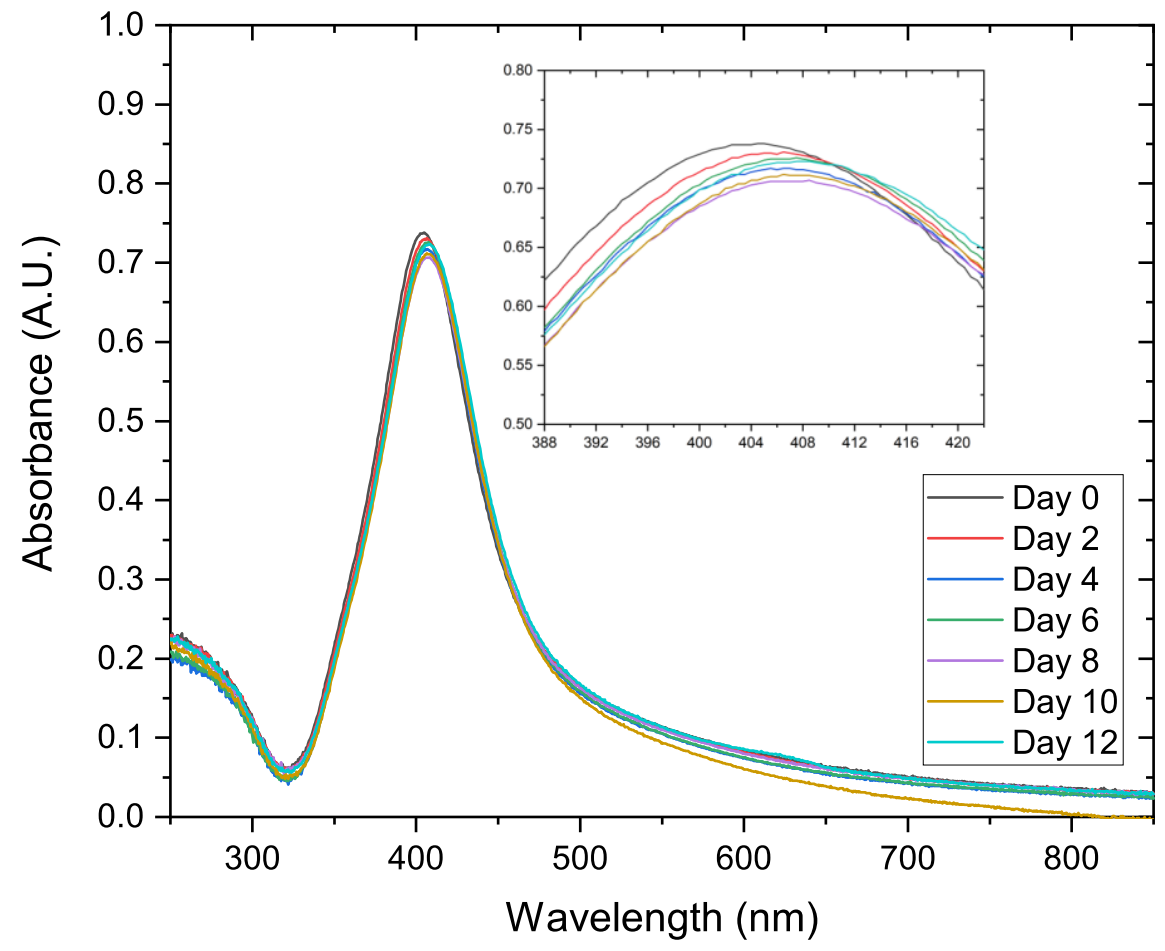

Figure 5: Absorption spectrum of $\mathrm{AgNps}$ produced in a weak $\mathrm{NaCl}$ ionic solvent of 0.86 $\mathrm{mg} / \mathrm{L}$. The plasmonic peak was at $404 \mathrm{~nm}$ at day 0 , and red shifted to $407 \mathrm{~nm}$ at day 12 . The signal intensity also dropped by $4.3 \%$.

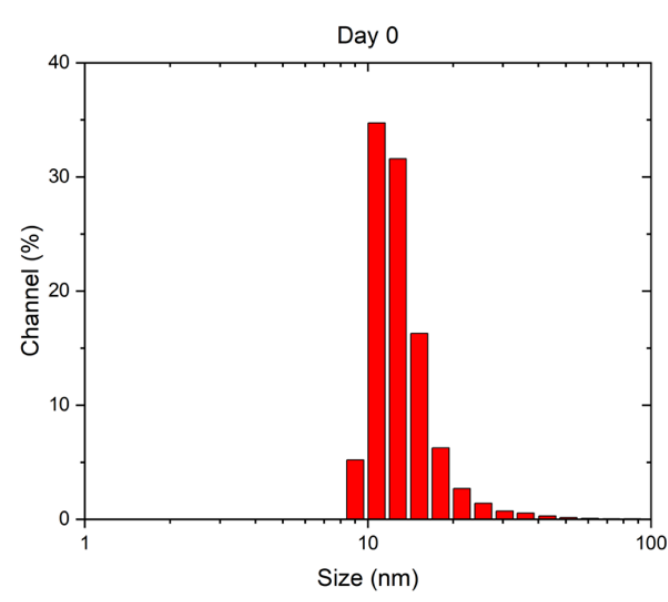

(a)

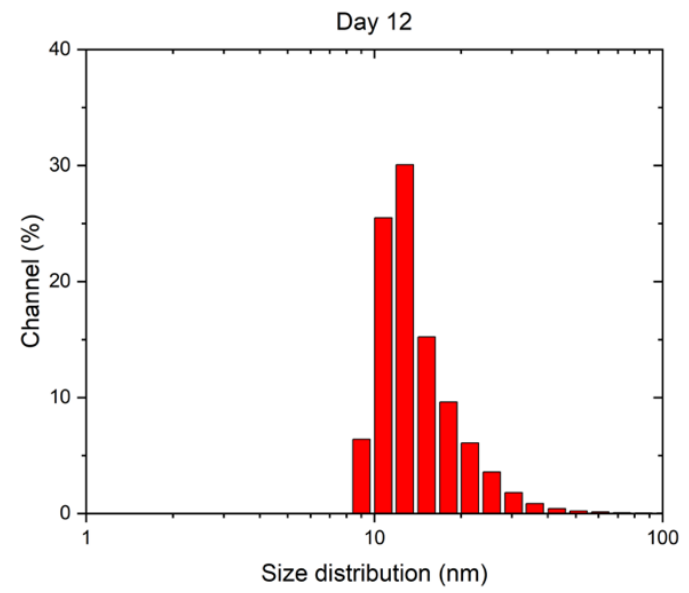

(b) 
Figure 6: DLS measurement of AgNps produced in the presence of $0.86 \mathrm{mg} / \mathrm{L} \mathrm{NaCl} / \mathrm{DI}$ water solution showing (a) the peak diameter of $11.3 \mathrm{~nm}$ and a width of $5 \mathrm{~nm}$ at day 0 , and (b) a peak diameter of $11.9 \mathrm{~nm}$ and an increased width of $7 \mathrm{~nm}$ at day 12 .

\section{Conclusions}

Silver nanoparticles were produced utilising a new laminar flow "semi-batch" production method. The colloids were seen to be comparable with commercially available colloids in terms of optical response, size and stability. The colloids were produced within a 15 minute period and could be directly applied to thin-film printing of anti-microbial surfaces. If applied for the printing of conductive circuits, post colloid production processing would be required to increase their concentration. Using a weak ionic liquid for Nps production was found to provide size quenching during providing a pathway towards increased size control during the production process. However, it was seen to have a more variable effect on colloid stability than when DI water alone was used as the solvent. Electrolyte addition along with its interaction with Nps concentration at the ablation site needs to be studied in more detail to gain a better understanding of maintaining high density, stable colloids. This work confirms that LASiS is a feasible technique towards commercial low-powered laser based nano-colloid production for thin-films manufacture.

\section{Acknowledgements}

This publication has emanated from research supported the School of Biotechnology, Dublin City University and by research grants from Science Foundation Ireland (SFI) under Grant Numbers 12/IA/1576 and 16/RC/3872 and is co-funded under the European Regional Development Fund and by I-Form industry partners. The Nanoflex in-situ particle size analyser was provided on trial by Microtrac Ltd. OSL group.

\section{References}

1. Calderón-Jiménez B, Johnson ME, Montoro Bustos AR, Murphy KE, Winchester MR, Vega Baudrit JR. Silver Nanoparticles: Technological Advances, Societal Impacts, and Metrological Challenges. Front Chem. 2017;5(February):1-26.

2. Cao L, Bai X, Lin Z, Zhang P, Deng S, Du X, et al. The preparation of Ag nanoparticle and ink used for inkjet printing of paper based conductive patterns. Materials (Basel). 2017;10(9).

3. Qing Y, Cheng L, Li R, Liu G, Zhang Y, Tang X, et al. Potential antibacterial mechanism of silver nanoparticles and the optimization of orthopedic implants by advanced modification technologies. Int J Nanomedicine. 2018;13:3311-27.

4. Lee SH, Jun BH. Silver nanoparticles: Synthesis and application for nanomedicine. Int J Mol Sci. 2019;20(4).

5. Rehbock C, Jakobi J, Gamrad L, van der Meer S, Tiedemann D, Taylor U, et al. Current state of laser synthesis of metal and alloy nanoparticles as ligand-free reference materials for nanotoxicological assays. Beilstein J Nanotechnol. 2014;5(1).

6. Intartaglia R, Barchanski A, Bagga K, Genovese A, Das G, Wagener P, et al. Bioconjugated silicon quantum dots from one-step green synthesis. Nanoscale. 2012;4(4):1271-4.

7. Dzhafarov TD, Pashaev AM, Tagiev BG, Aslanov SS, Ragimov SH, Aliev AA. Influence of silver nanoparticles on the photovoltaic parameters of silicon solar cells. Adv nano Res. 2016;3(3):133-41.

8. Campos-cuerva C, Zieba M, Sebastian V. Screen-printed nanoparticles as anti- counterfeiting tags. 2016;

9. Lee KS, El-Sayed MA. Gold and silver nanoparticles in sensing and imaging: Sensitivity of 
plasmon response to size, shape, and metal composition. J Phys Chem B. 2006;110(39):192205 .

10. Panáček A, Kolář M, Večeřová R, Prucek R, Soukupová J, Kryštof V, et al. Antifungal activity of silver nanoparticles against Candida spp. Biomaterials. 2009;30(31):6333-40.

11. Gao M, Sun L, Wang Z, Zhao Y. Controlled synthesis of Ag nanoparticles with different morphologies and their antibacterial properties. Mater Sci Eng C [Internet]. 2013 Jan 1 [cited 2018 Nov 7];33(1):397-404. Available from: https://www.sciencedirect.com/science/article/pii/S0928493112004377

12. Lee D, Cohen RE, Rubner MF. Antibacterial properties of Ag nanoparticle loaded multilayers and formation of magnetically directed antibacterial microparticles. Langmuir. 2005;21(21):9651-9.

13. Carbone M, Donia DT, Sabbatella G, Antiochia R. Silver nanoparticles in polymeric matrices for fresh food packaging. J King Saud Univ - Sci [Internet]. 2016;28(4):273-9. Available from: http://dx.doi.org/10.1016/j.jksus.2016.05.004

14. Chien Dang M, Dung Dang TM, Fribourg-Blanc E. Silver nanoparticles ink synthesis for conductive patterns fabrication using inkjet printing technology. Adv Nat Sci Nanosci Nanotechnol. 2015;6(1).

15. Raut NC, Al-Shamery K. Inkjet printing metals on flexible materials for plastic and paper electronics. J Mater Chem C. 2018;6(7):1618-41.

16. Zhang D, Gökce B, Barcikowski S. Laser Synthesis and Processing of Colloids: Fundamentals and Applications. Chem Rev. 2017;117(5):3990-4103.

17. Wagener P, Barcikowski S, Dell'Aglio M, Kohsakowski S, Santagata A, Gökce B, et al. High productive and continuous nanoparticle fabrication by laser ablation of a wire-target in a liquid jet. Appl Surf Sci [Internet]. 2017;403:487-99. Available from: http://dx.doi.org/10.1016/j.apsusc.2017.01.077

18. Nachev P, van 'T Zand DD, Coger V, Wagener P, Reimers K, Vogt PM, et al. Synthesis of hybrid microgels by coupling of laser ablation and polymerization in aqueous medium. J Laser Appl. 2012;24(4):042012.

19. Mafuné, F, Kohno J, Takeda Y, Kondow T, Sawabe H. Structure and Stability of Silver Nanoparticles in Aqueous Solution Produced by Laser Ablation. J Phys Chem B. 2002;104(35):8333-7.

20. Jeon J-W, Yoon S, Choi H, Kim J, Farson D, Cho S-H. The Effect of Laser Pulse Widths on Laser-Ag Nanoparticle Interaction: Femto- to Nanosecond Lasers. Appl Sci. 2018;8(1):112.

21. Barcikowski S, Amendola V, Marzun G, Rehbock C, Reichenberger S, Zhang D, et al. Handbook of Laser Synthesis of Colloids. 2016;

22. Valenti LE, Giacomelli CE. Stability of silver nanoparticles: agglomeration and oxidation in biological relevant conditions. J Nanoparticle Res. 2017;19(5).

23. Mahmoodi A, Shoorshinie SZ, Dorranian D. Synthesis and characterization of $\mathrm{AgCl}$ nanoparticles produced by laser ablation of $\mathrm{Ag}$ in $\mathrm{NaCl}$ solution. Appl Phys A Mater Sci Process. 2016;122(4):1-10.

24. Letzel A, Gökce B, Wagener P, Ibrahimkutty S, Menzel A, Plech A, et al. Size Quenching during Laser Synthesis of Colloids Happens Already in the Vapor Phase of the Cavitation Bubble. J Phys Chem C. 2017;121(9):5356-65.

25. Rehbock C, Merk V, Gamrad L, Streubel R, Barcikowski S. Size control of laser-fabricated surfactant-free gold nanoparticles with highly diluted electrolytes and their subsequent bioconjugation. Phys Chem Chem Phys [Internet]. 2013;15(9):3057-67. Available from: http://pubs.rsc.org/en/content/articlehtml/2013/cp/c2cp42641b

26. Barcikowski S, Amendola V, Marzun G, Rehbock C. Handbook of Laser Sintheshis. 2016;(April). 